Neural, Parallel, and Scientific Computations 28 (2020) 73-82

\title{
MACHINE LEARNING DATA DETECTION POISONING ATTACKS USING RESOURCE SCHEMES MULTI-LINEAR REGRESSION
}

\author{
KAVITHA GANESAN \\ Department of Electronics and Communication Engineering \\ Government College of Technology, Salem, India \\ Email : kavitha@gcesalem.edu.in
}

\begin{abstract}
Machine learning methods become more and more popular. The purpose of using these methods is to become more and more popular network security components such as firewalls and anti-virus software such as machine learning methods expected to rise. Data machine learning systems provided by well-trained users can be vulnerable to attacks, poisoning data where malicious users inject fake training data, and damage the learning model. Data poisoning attacks can damage the integrity of the machine learning model by introducing malicious training models that affect results during testing. Distributed machine learning (DML) and Semi-DML is training that can be realized from a large database when any node is able to work out accurate results at an acceptable time. Compared to this inevitably diverse environment the attack will still expose potential targets. In this proposed method, we introduced the method for data detection poisoning, Data Poison Detection Program, Resource Schemes Multi-Linear Regression (RSMLR) to provide better learning protection and assistance from central sources. Proper allocation of resources in RSMLR can reduce resource waste. The application of modifying the data poison detection program can extend the system even more dynamically according to the environment and attack intensity. In addition, many of the components will increase the resource consumption of the system due to training.
\end{abstract}

Key words: Machine learning, Data detection, poisoning scheme, Resource Schemes MultiLinear Regression (RSMLR), Distributed machine learning (DML) and Semi-DML.

\section{Introduction}

System security tries develop a sensible cutoff between the structure and the outside world to ensure the reliability of the structure against ambush. Simulated intelligence, nevertheless, was readied dependent on information got clearly from space by all the hugest materials. Especially arranged customer data systems aggressors can't simply mix malevolent data by making a customer account. Hurting attacks system requires data like us to reevaluate what decision techniques being secured. Data poison attacks are security risks acknowledged by AI models during the planning stage. Deep Neural Networks (DNNs) resources require a lot of data planning and computational and complex task showing. In various realistic conditions, it is significant, to summarize outside articles or assemble them from re-appropriated model planning. There is a veritable security challenge that these AI systems can provoke pollution. Poison attacks were investigated because of PC beginning stage excusal, picture extraordinary framework rivals organization sense examination, and malware area. Hurting against data protection frameworks as often as possible twists around the acknowledgment of eccentricities though various procedures. More occurrences of hurting, which are ordinarily traces of the mind boggling loss of lives and property of the

Received March 11, 2020

www.dynamicpublishers.org
1061-5369 \$15.00 @ Dynamic Publishers, Inc. https://doi.org/10.46719/NPSC20202821 
people. In order to deal with these conditions, the fundamental task, and paying little mind to what the occupation, lab, or time, is the area of the sort of poison that has not been made plans to deal with the subsequent hurting setback.

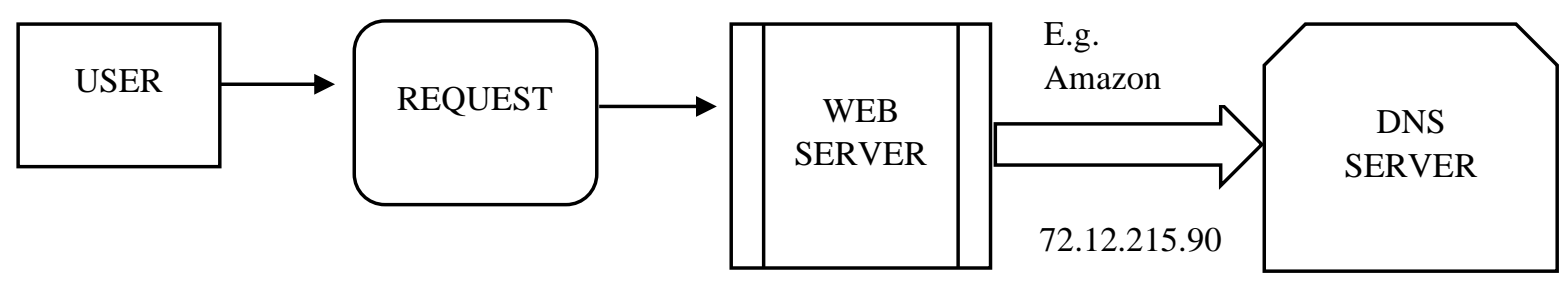

Figure 1: DNS Poisoning Attacks

Figure 1 describe that, User can request the web server, the server accept the request for the web site address to the DNS server.DNS poisoning attacks remove the unwanted files or wastage resources.

Thus, it can viably build up a harming innovation in this way improving the division's capacity to manage harming cases, enhancing screen preparing nearby handling abilities and lab harming, to explain harming dependent on the casualty's toxic substance side effects. Strife AI endeavors have been abused to depict AI, which misleads innovation utilized in the field of car models by entering any preparation time or end time. The AI technique for managing them is separated into two sorts relying upon the hour of the assault: Sample Training Poison Data Pre: The assailant learns a few marks of the preparation informational index before the shape changes. Information creation depends on the able model: the model wherein the assailant's powers are prepared to deliver the real yield information for turnaround activity. Assaults are extremely risky thinking about the results and effect of these two kinds. When seeing business AI items, you can see that information harming assaults represent a more noteworthy danger. Practically all the preparation information required for business items are set up from the establishment framework. Deliver, it tends to be said that these datasets are anything but difficult to harm.

\section{Related work}

Artificial intelligence is a clear probabilistic request subject to Bayes' theoretical application. Adjusting guiltlessly empowers the Bayes request by expecting that the credit should be given to a class self-sufficiently. With this reasonable assumption, the discretionary Bayes classifier is the most bewildering arranged competition. It will give generous results with the properties of the data. The most extraordinary outline is a posteriori (map) hypothesis got together with past data on hypothesis appraisal attributes. In a general sense, the probability model is a fundamental probability scattering subject to the course of action brand name performed. The subjective inclination request of the logical order wind up being more compelling than the standard based tree appendage structure and procedure. One of the essential focal points of the unpredictable Bayes classifier is the restricted amount of data that is then arranged to complete the portrayal learning limits requirements. Discretionary inclination course of action request is best gotten done with this instructional exercise. They decline the opportunity of sham choices, they are called better gathering. The gullible Bayes classifier request is an astounding procedure to describe the best execution with respect to exactness is to use the prohibitive probability of the unequivocally of a particular class to research the unpredictable Bayes classifier. Since discretionary Bayes logical classification has a speedy enrollment of learning, such course of action is one of the two rule kinds of Bernoulli's model with reference strategy factors as model benchmarks. 
ADNN preparing is done in an unexpected manner in comparison to different techniques. The preparation input model is partitioned into two sections. One of the two pieces of the preparation input is to figure out how to the neural system in the preparation mode it expects to change its loads. At the point when the second piece of the preparation section is given, each record of ADNN will be checked. On the off chance that the record is as of now a certified technique, the ADNN arrangement is the comparing class input. In the event that the record is new, ADNN attempts to incorporate with any previously learned class. On the off chance that the grouping is fruitful, the new record will be added to the preparation bundle to additionally characterize the record. Something else, the concealed hubs are effectively ordered as a number in learning wages up to advances and records. Thusly, the arrangement wrap is set up by ADNN to totally portray steady data. Of these, these movements occurred for the PARAMETER_INDEX stretch [0.20, 0.90]. In the wake of testing the speed estimation of a couple of models was fixed at 0.40 . The learning rate is adjusted over the range $[0.10,0.50]$ and a while later changed as per over 0.30 . Regardless, different relationship over the framework will be pushed at estimated timeframes [-0, 50, 0.50]. In the instructional exercise, the estimation used by ADNN graphs the nine instructional exercise limits in a little way. A particularly humble number of ages gather in the adaptable adaptability of 1000, in spite of the way that the gathering accuracy is done rapidly and the adaptable diffraction is assessed so the intentional advancement is too high to even think about evening consider changing the district incline. The test is thusly eluted with a quantifiable sub-slant.

\section{Materials and Method}

Computer based intelligence methods are used to adequately recognize the intercession used to bunch continuous data. Right when ML is properly arranged and realized, it deals with the issues looked by real and rule-based strategies. The proposed technique can be used to recognize tremendous quantifiable changes in the utilization of the endeavor resource diverse backslide (RSMLR), a structure used to choose to arrange system unconventionalities. By virtue of gaining extraordinary resources, progressing data gave through getting ready and testing are properly assembled to address different kinds of attacks and run of the mill classes. In the RSMLR circumstance, the Center has a subcommittee of planning task data poison acknowledgment programs with no unnecessary figuring resource

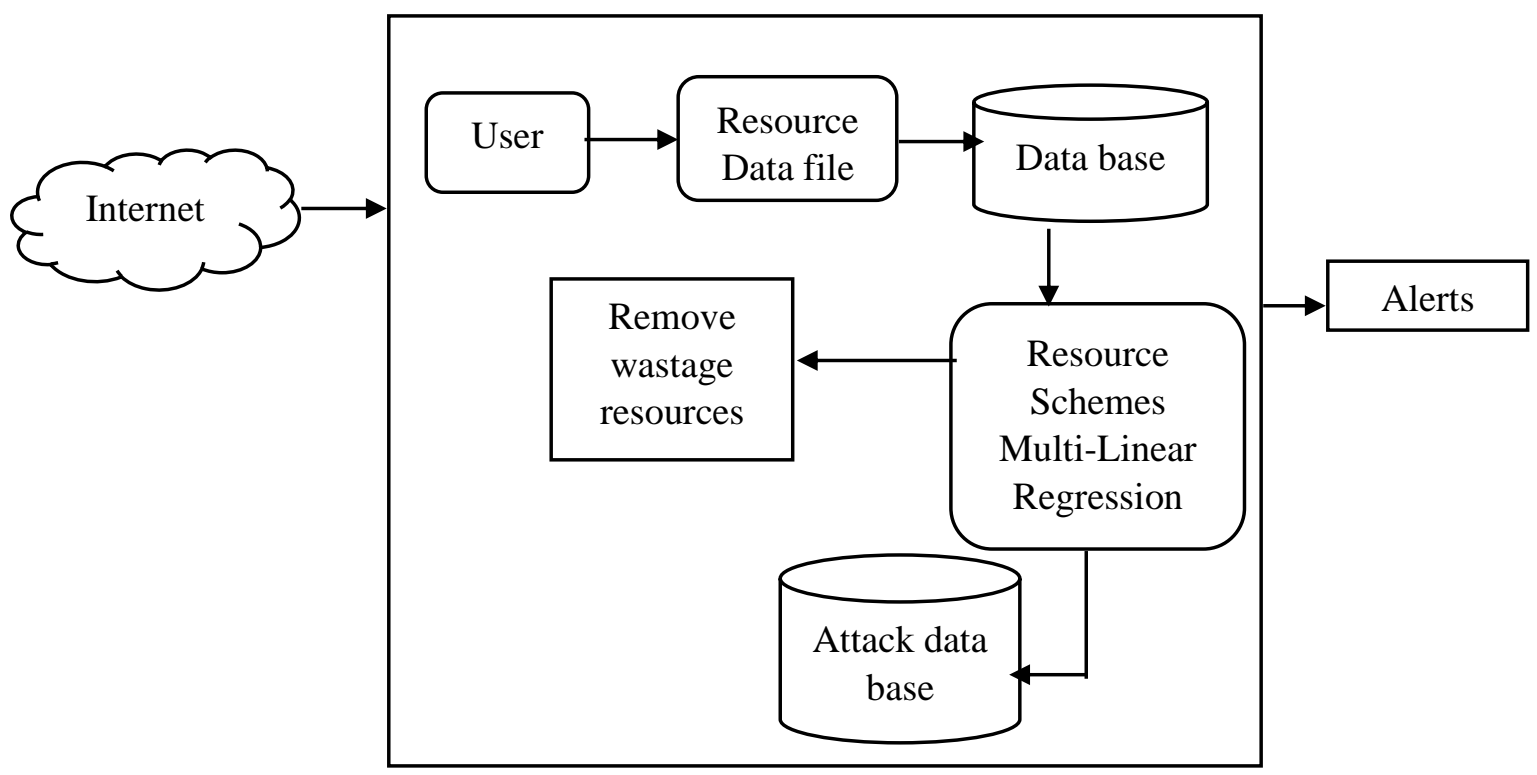

Figure 2: Block diagram 
sharing. For this circumstance, the center just arranges the planning. Data Poison Detection Program to give better taking in security and help from central sources. If structure resources are to be used effectively, the best resource appropriation system is Development. The RSMLR program can in a general sense improve precision. Resources can constrain the wastage of explicit fundamental resources of the data poison area program.

Figure 2 describes the, User input instructions find and attack information store, remove unwanted resources to remove wasted resources, finally detect alert messages to display information data source file.

\subsection{Attack Multilinear Regression model}

Dynamic Input instructive assortment is given to Attack Multilinear Regression. A couple of straight backslides of scattering ambushes was used in this examination. In a general sense, AMR covers the depiction of yield resources by setting the information. Every benefit and each archive is associated with all sources. Every association has a relating weight. It goes over the model in the planning data record of all possible hurting ambushes, and it is obviously hard to choose the most fitting response for the hurting attack. The issue here is missing the mark on hurting attack game plan and model retraining time. Evaluating new model lead quickly, and flexibility is key in endeavoring to deal with this issue. In this article, we have developed a closed structure answer for evaluating another technique in the wake of hurting without modifying themselves.

\section{Algorithm}

Input: Training value model, Target value $\{X, Y\}$

Output: Classification values

The number of boot input and output files and resources Training Value

Start.

Within starting weights and limits

Current training situation

Divide it into two resource files.

Consider the first file as a group

Predicting hidden and activating output resources

Continue to change weights until the crowd.

Complete learning assigns it to the real class

Notice the files in the second set

Once the system is known the resource file

Go to 7 , then specify the class

-Otherwise

End

Training data $\{\mathrm{X}, \mathrm{Y}\}$, where $\mathrm{X}$ joins date and time, temperature, and $\mathrm{Y}$ load are illustrated. Because, using an outstanding and precise model, talk about the way that the store data in Attack Y is undefined, and a while later the two cases are significant in view of the logical properties of the two resource records that are executed by the elements that depict the reason behind the parcel of $\mathrm{Y}$ and the load data to ambush the two data in $\mathrm{X}$. Vacillate in size.

\subsection{Data Poisoning Detection Attack}

In a Data area hurting ambush, the understudy planning data that impacts the learning computation work is set by explicit targets described by the threatening to sprinkle the adversary in the model, the counter-plan of the model grows the error of the learning estimation, yet more honest destinations can be seen as further. Most of the hurting attack 
lines, we simply consider the twofold straight portrayal issue here. Different degrees of attack data, regardless of the way that can diminish our chances of being acknowledged, have a profound comprehension of the right data Wastage target system: both Learning figuring (tallying limit assurance limit) and data arrangement.

\section{Algorithm}

Procedure POISONING (STR, Sval, $, 2, \lambda, x)$

$$
\begin{aligned}
& \mathrm{T} \leftarrow 0 \\
& \quad \mathrm{~S}(\mathrm{t}) \mathrm{p}=\{\mathrm{a}(\mathrm{t}) \mathrm{bj}, \mathrm{cpj}\} \mathrm{xj}=1 \leftarrow \text { choose Initial points }(\mathrm{STR})
\end{aligned}
$$

Repeat

$$
\begin{aligned}
& \mathrm{S}^{\wedge} \mathrm{a}=\{\mathrm{axj}, \mathrm{ybj}\} \mathrm{xj}=1 \leftarrow \mathrm{S}(\mathrm{t}) \mathrm{a} \\
& \text { For } \mathrm{j}=1 \text {, } \mathrm{x} \text { do } \\
& (\mathrm{Z}, \mathrm{y}) \leftarrow \text { Poisoning line (STR } \cup \mathrm{S} \wedge a) \\
& \text { Compute } \Delta(\mathrm{apj})=\partial \mathrm{OA} / \partial \mathrm{bpj}) \\
& \mathrm{H}=\Pi X(\mathrm{xpj}+\Delta(\mathrm{xpj}))-\mathrm{xpj} \\
& \eta \leftarrow \text { HS (STR, Sval, S^a, h) } \\
& \mathrm{T} \leftarrow \mathrm{t}+1 \\
& A p j \leftarrow \text { apj }+\eta \text { hT } \\
& \mathrm{S}(\mathrm{t}) \mathrm{p}=\{\mathrm{a}(\mathrm{t}) \mathrm{pj}, \mathrm{ypj}\} \mathrm{xj}=1 \leftarrow \mathrm{S}^{\wedge} \mathrm{a} \\
& \text { Until }|\mathrm{OA}(\mathrm{S}(\mathrm{t}) \mathrm{p})-\mathrm{OA}(\mathrm{S}(\mathrm{t}-1) \mathrm{p})|<\mathrm{q}
\end{aligned}
$$

Return

Where, STR-Initial values, Data detection is the best poison attack strategy classification process. Algorithm using the proposed attack technique, some of the algorithms we have described below have been modified. Poison B for example that starts the boot process wants the attacker to detection point.

\subsection{Resource Schemes Multi-Linear Regression (RSMLR)}

This system is reiterated for each class in the enlightening record. The class will be named reliant on the most raised probability regard. Resource plan application has a spot with a specific arrangement. For example, unique direct backslide (RSMLR), which is used to investigate misalignment using the opposite probability of area, is realized by a twofold exercise vector request module and an impostor or an attack set up to exhibit the probability of an ambush being foreseen high bore. The errand of class request modules relies upon a high probability regard. One of the standard focal points is that this report doesn't see the misrepresentation level as outstandingly low according to the request delineated. It works outstandingly when data centers are viewed as for all intents and purposes.

\section{Algorithm}

Input: Training value, Target value

Output: Removes wastage resource

Step 1. Read the input file

Step 2. Complete RSMLR training values

Step 3. Analysis the Current test status

Step 4. for each test feature vector

Step 5. Calculations of training models are the number belonging to a particular class

Step 6. Calculated Attribute Value a certain class of work is often classified as a specific achievement

Step 7. Make all records in the process

Step 8.End

Where RSMLR- Resource Schemes Multi-Linear Regression In the algorithm steps find the training and test values for the resources, RSMLR is removed the wastage values form the process. 


\section{Result and discussion}

The results of the Resource classification are discussed in this section. Structure and multiple linear classifiers and multiple linear model RSMLR results outperformed the best simple resource scheme. In the proposed method of RSMLR has made significant progress in comparing previous method of DML performance and semi-DML.

Table 1: Simulation Parameters

\begin{tabular}{|c|c|}
\hline Parameters & Value \\
\hline Simulation Tool & python \\
\hline Data size & $100 \mathrm{mb}$ \\
\hline Transferring files & 500 \\
\hline Resource & Resource scheme Multiple linear \\
& regression \\
\hline Detection & Detection Data poisoning \\
\hline
\end{tabular}

Table 1 shows the, Resource Detection Technique Measures, Attacks Poison Data Resource Project Multiple linear regression method Detects attacks from resources using our proposed method.

\subsection{Analysis the Detection Accuracy}

Classification accuracy is simply the correct classification ratio, whether it is grouped in a single experiment or there is some variation in the idea of using crossvalidation. The algorithm can improve command class accuracy and improve the resolution of the problem without wasting resources.

Accuracy-TP+TN/TP+FP+TN+FN*100

Table 2: Detection Accuracy

\begin{tabular}{|r|r|r|r|}
\hline No.of files & DML & $\begin{array}{l}\text { SEMI } \\
\text { DML }\end{array}$ & RSMLR \\
\hline 20 & 75 & 80 & 88 \\
\hline 40 & 81 & 89 & 93 \\
\hline 60 & 85 & 88 & 95 \\
\hline 80 & 88 & 91 & 96 \\
\hline 100 & 90 & 92 & 97 \\
\hline
\end{tabular}

Analysis in Accurcy in \%

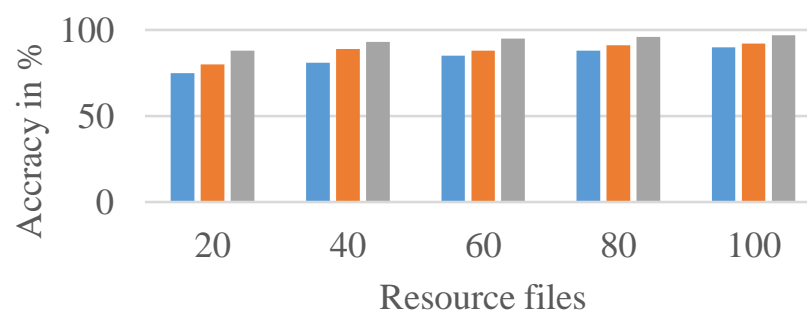

$\square \mathrm{DML} \quad \square$ SEMI DML $\quad$ RSMLR

Figure 3: Analysis of Detection Accuracy 
Figure 3shows that the observed detection accuracy performance of existing methods for DML is $90 \%$, and Semi-DML is $92 \%$. The proposed RSMLR implementation produces a higher efficiency of $97 \%$ improving the accuracy than other methods.

\subsection{Reducing Data Poisoning Attack}

Data poisoning attack affects the integrity of the resulting compromise at the time of testing by removing malicious training samples. In this task, the training data file wastes data by adding resources to reduce poisoning attack.

Table 3: Reducing Data Poisoning Attack

\begin{tabular}{|r|r|r|r|}
\hline No.of. Files & DML & $\begin{array}{l}\text { Semi- } \\
\text { DML }\end{array}$ & RSMLR \\
\hline 20 & 75 & 65 & 60 \\
\hline 40 & 73 & 68 & 55 \\
\hline 60 & 70 & 66 & 52 \\
\hline 80 & 68 & 58 & 48 \\
\hline 100 & 65 & 55 & 40 \\
\hline
\end{tabular}

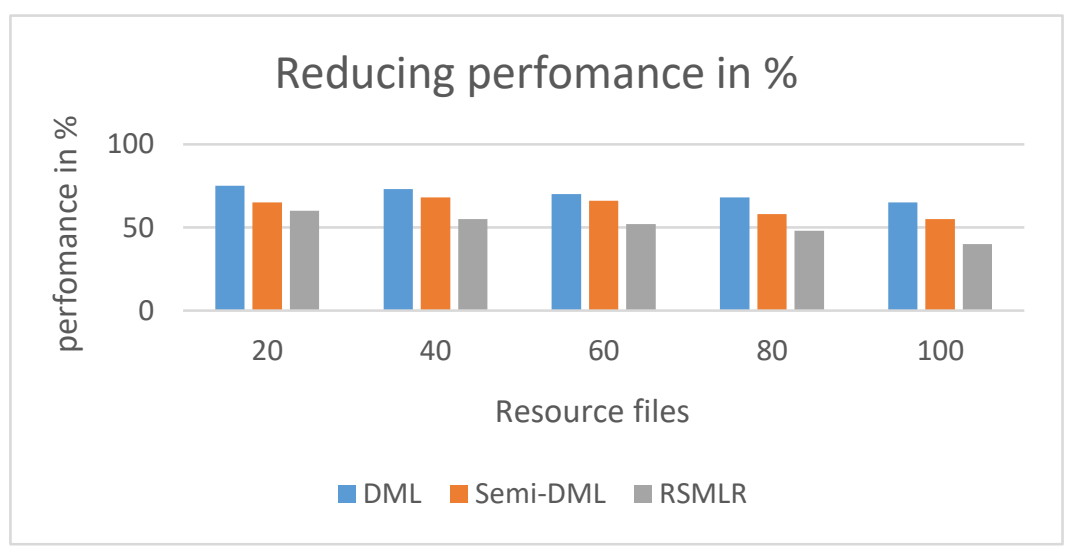

Figure 4: Reducing data detection attack

Figure 4 describes the, Reducing data detection performance for comparing of existing methods for DML in $65 \%$ Semi-DML in 55\% and the proposed method for RSMLR in $40 \%$ to reducing the data detection attack

\subsection{Time complexity}

Both were taken advantage of to minimize time change due to data transfer. Data on the network can potentially increase except for the RSMLR method used for encryption. Using RSMLR will eliminate wasted resources and reduce on-site time.

Table 5: Time complexity

\begin{tabular}{|r|r|r|r|}
\hline No.of. Files DML & & semi-SML & RSMLR \\
\hline 10 & 15 & 12 & 10 \\
\hline 20 & 25 & 22 & 20 \\
\hline 30 & 30 & 28 & 25 \\
\hline 40 & 40 & 32 & 25 \\
\hline 50 & 35 & 32 & 30 \\
\hline 60 & 45 & 40 & 28 \\
\hline
\end{tabular}




\section{TIME COMPLEXITY}

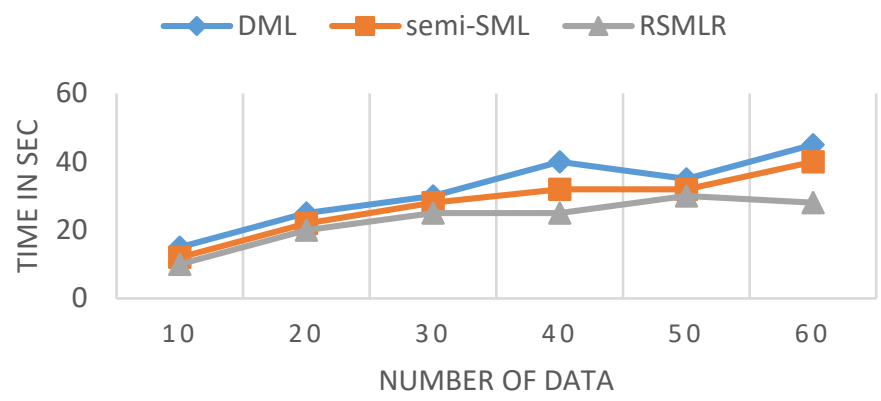

Figure 5: Time complexity

Figure 5 describes the delay performance is calculated based on the number of data Data to be delivered to the destination within a period of timeTime. It is a measure in this way. Comparing the previous time complexity decrease the proposed method. In the time complexity of the number of Data, DML calculating the timeline transfers the minimum of $100 \mathrm{mb}$ data size data sends in 45sec, Semi-DML 40sec, and RSMLR in 28sec.In the proposed method reduces the time complexity.

\subsection{Analysis the Failure Rate}

Evaluate failure performance and obtain these requirements in the development process to ensure that they do not bring complex failure situations or requirements. Identification design features facilitate error detection and reduce the spread of failures throughout the data processing transaction. Develop software testing programs and algorithms dedicated to software behavior related to motion and fault detection, isolation and recovery.

Table 5:Failed Rate Detection

\begin{tabular}{|r|r|l|l|}
\hline No.of. Files & DML & $\begin{array}{l}\text { Semi- } \\
\text { DML }\end{array}$ & RSMLR \\
\hline 20 & 85 & 75 & 68 \\
\hline 40 & 80 & 68 & 66 \\
\hline 60 & 75 & 65 & 58 \\
\hline 80 & 68 & 58 & 55 \\
\hline 100 & 62 & 55 & 50 \\
\hline
\end{tabular}

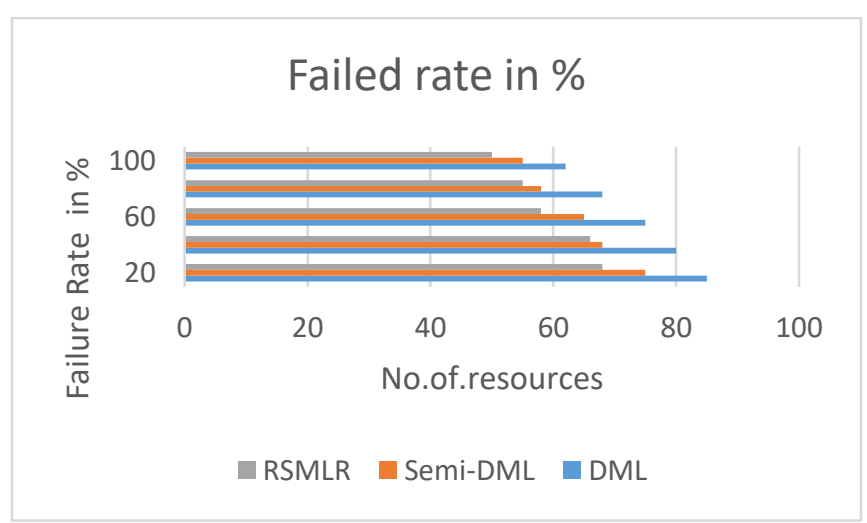

Figure 6 : Analysis of Failed Rate 
Figure 6 describes the, analyzing failed data detection performance for comparing of existing methods for DML in $62 \%$ Semi-DML in $55 \%$ and the proposed method for RSMLR in $50 \%$ to reducing the data failed attack.

\section{Conclusion}

Poisoning attacks are considered one of the most relevant new threats to machine learning and data-based technologies. Since many applications rely on unreliable data collection in the wild, attackers can focus on malicious data or blindly reduce system performance. Attacks by Data Poison Training Database Poison Machine Learning System to pose a major security threat. Attack on RSMLR algorithm is proposed and our attack proved to be different security algorithms and machine learning capability. The ultimate goal in the analysis of poison attacks is to develop defensive strategies. Experimental results suggest that there is a trade-off between first attack and detective ability. Therefore, the method of action may be a potential defense against detecting the behavior of resources without wasting accuracy, and using practical learning methods.

\section{References}

1 Mohan Li ; Yanbin Sun ; Hui Lu ; Sabita Maharjan ; Zhihong Tian, "Deep Reinforcement Learning for Partially Observable Data Poisoning Attack in Crowdsensing Systems", IEEE Internet of Things Journal ( Volume: 7 , Issue: 7 , July 2020 ).

2 Juncheng Shen ; Xiaolei Zhu ; De Ma, "TensorClog: An Imperceptible Poisoning Attack on Deep Neural Network Applications", IEEE Access ( Volume: 7 2019).

3 Ping Zhao ; Haojun Huang ; Xiaohui Zhao ; Daiyu Huang, "P3: Privacy-Preserving Scheme Against Poisoning Attacks in Mobile-Edge Computing", IEEE Transactions on Computational Social Systems ( Volume: 7 , Issue: 3 , June 2020 ).

4 M. Shayan, C. Fung, C. J. Yoon, and I. Beschastnikh, "Biscotti: A ledgerfor private and secure peer-to-peer machine learning," arXiv preprintarXiv:1811.09904, 2018.

5 Yijin Chen ; Yuming Mao ; Haoyang Liang ; Shui Yu ; Yunkai Wei ; Supeng Leng, "Data Poison Detection Schemes for Distributed Machine Learning", IEEE Access (Volume: 8 2019).

6 Lingchen Zhao ; Shengshan Hu ; Qian Wang ; Jianlin Jiang ; Shen Chao ; Xiangyang Luo; Pengfei Hu, "Shielding Collaborative Learning: Mitigating Poisoning Attacks through Client-Side Detection", IEEE Transactions on Dependable and Secure Computing ( Early Access 2020).

7 Xiaoyan Hu ; Jian Gong ; Guang Cheng ; Guoqiang Zhang ; Chengyu Fan, "Mitigating Content Poisoning With Name-Key Based Forwarding and Multipath Forwarding Based Inband Probe for Energy Management in Smart Cities", IEEE Access ( Volume: 6 2018).

8 Wenbo Jiang ; Hongwei Li ; Sen Liu ; Xizhao Luo ; Rongxing Lu, "Poisoning and Evasion Attacks Against Deep Learning Algorithms in Autonomous Vehicles", IEEE Transactions on Vehicular Technology ( Volume: 69 , Issue: 4, April 2020 ).

9 Jiayin Zhu ; Xuehua Zhao ; Huaizhong Li ; Huiling Chen ; Gang Wu, "An Effective Machine Learning Approach for Identifying the Glyphosate Poisoning Status in Rats Using Blood Routine Test", IEEE Access ( Volume: 6 2018). 
10 N. Baracaldo, B. Chen, H. Ludwig, and J. A. Safavi, "Mitigatingpoisoning attacks on machine learning models: A data provenance basedapproach," in Proc. of AISec'17. ACM, 2017, pp. 103-110.

11 Yalin Sagduyu ; Yi Shi ; Tugba Erpek, "Adversarial Deep Learning for Over-the-Air Spectrum Poisoning Attacks", IEEE Transactions on Mobile Computing ( Early Access 2019).

12 Jiadai Wang; Yawen Tan ; Jiajia Liu ; Yanning Zhang, "Topology Poisoning Attack in SDN-enabled Vehicular Edge Network", IEEE Internet of Things Journal ( Early Access 2020).

13 Qianlong Wang; Yifan Guo ; Lixing Yu ; Xuhui Chen ; Pan Li, "Deep Q-Network based Feature Selection for Multi-Sourced Data Cleaning", IEEE Internet of Things Journal ( Early Access 2020).

14 Qi Li ; Patrick P. C. Lee ; Peng Zhang ; Purui Su ; Liang He ; Kui Ren, "CapabilityBased Security Enforcement in Named Data Networking", IEEE/ACM Transactions on Networking ( Volume: 25 , Issue: 5 , Oct. 2017 ).

15 T. Gu, B. Dolan-Gavitt, and S. Garg, "Badnets: Identifying vulnerabilities in the machine learning model supply chain,” arXiv preprint arXiv:1708.06733, 2017.

16 N. Baracaldo, B. Chen, H. Ludwig, and J. A. Safavi, "Mitigating poisoning attacks on machine learning models: A data provenance based approach," in Proc. of AISec'17. ACM, 2017, pp. 103-10.

17 S. Chen et al., "Automated poisoning attacks and defenses in malware detection systems: An adversarial machine learning approach," Comput.Secur., vol. 73, pp. 326-344, Mar. 2018.

18 T. Chen, M. Li, Y. Li, M. Lin, N. Wang, M. Wang, T. Xiao, B. Xu,C. Zhang, and Z. Zhang, "Mxnet: A flexible and efficient machine learning library for heterogeneous distributed systems," CoRR, vol.abs/1512.01274, 2015.

19 P. Blanchard, R. Guerraoui, J. Stainer et al., "Machine learning with adversaries: Byzantine tolerant gradient descent," in Proc. of NeurIPS'17,2017, pp. 119-129.

20.Z. Yin, F. Wang, W. Liu, and S. Chawla, "Sparse feature attacks in adversarial learning," IEEE Transactions on Knowledge and Data Engineering, vol. 30, no. 6, pp. 1164-1177, 2018. 